\title{
Chemical properties of transactinides
}

\author{
H.W. Gäggeler ${ }^{a}$ \\ Paul Scherrer Institut, 5232 Villigen, Switzerland and University of Bern, Freiestrasse 3, 3012 Bern, Switzerland
}

Received: 10 December 2004 / Revised version: 4 April 2005 /

Published online: 15 August 2005 - C Società Italiana di Fisica / Springer-Verlag 2005

\begin{abstract}
First investigations of chemical properties of bohrium $(Z=107)$ and hassium $(Z=108)$ showed an expected behaviour as ordinary members of groups 7 and 8 of the periodic table. Two attempts to study element 112 yielded some indication for a behaviour like a very volatile noble metal. However, a very recent experiment to confirm this preliminary observation failed. Two examples are described how chemical studies may help to support element discovery claims from purely physics experiments. The two examples are the discovery claims of the elements 112 and 115, respectively, where the progenies hassium and dubnium were chemically identified.
\end{abstract}

\section{Introduction}

During the last few decades the discovery of new elements changed from a field of chemistry to a topic in nuclear physics. The last example of a chemical contribution to an element discovery reaches back to the 1960s. A combination of physical and gas chemical experiments was considered sufficiently convincing by the International Union of Pure and Applied Chemistry (IUPAC) to approve the discovery of the first transactinide rutherfordium (Rf). For the elements up to seaborgium $(\mathrm{Sg})$ gas-jet techniques coupled to semiconductor detector systems were applied for their identification. The even heavier elements were discovered using the velocity SHIP at GSI (elements bohrium (Bh) through 112) and a gas-filled magnetic separator at FLNR in Dubna (elements 113-116 and 118) [1,2]. Element 113 has also been discovered independently at RIKEN [3]. However, the heaviest element that has been approved by IUPAC has the atomic number 111 (roentgenium, $\mathrm{Rg}$ ).

In the discovery experiments of the elements Bh through Rg, their $\alpha$-decay chains had an overlap with already known nuclides. This enabled an unequivocal identification of the new element. In the discovery experiment of element $112[4,5]$ a member of the decay chain $\left({ }^{261} \mathrm{Rf}\right)$ revealed a hitherto unknown decay property. Therefore, as yet, IUPAC has not approved the discovery of this element. This holds also for the recent discovery claims of elements 113-116 and 118 of the FLNR in Dubna [2,6]. These elements were produced in complete fusion reactions of ${ }^{48} \mathrm{Ca}$ beams with actinide targets. The observed decay chains all end in unknown final nuclides, mostly decaying by spontaneous fission (SF). Hence, not only are the primary evaporation residues unknown but also all

a Conference presenter; e-mail: heinz.gaeggeler@psi.ch members of the decay chains. For the assignments to a given element purely nuclear physics arguments are used (calculated excitation functions, decay properties of observed nuclei, cross bombardments etc.).

For a chemist the mere proof of the existence of a nucleus with a given number of protons is only part of a discovery of a new chemical element. As a member of the periodic table this new member has to find its place in this table, by no means a trivial task! The reasons are relativistic effects that might heavily influence chemical properties. Due to the high Coulomb force between the nucleus and orbiting electrons, energies of the atomic levels can be significantly changed. Symmetrical $s$ and $p_{1 / 2}$ orbitals have higher binding energies due to an increased overlap of the wave functions with the nucleus (direct relativistic effect). This causes a shrinking of the orbitals and screens, i.e. destabilizes high angular-momentum levels $\left(p_{3 / 2}, d, f\right)$ (indirect relativistic effects). As a consequence, the sequence of electron orbitals may deviate from "classical" expectation which in turn may cause an unexpected chemical behaviour.

During the last 5 years two elements have been investigated chemically for the first time: bohrium (Bh) [7, 8] and hassium $(\mathrm{Hs})[8,9]$. Currently, experiments are in progress to chemically identify element 112 [10,11]. In all these studies continuous gas phase techniques were applied that have proven to be fast and efficient.

Bohrium was separated in an isothermal gas chromatography experiment using the On-Line Gas Chemistry Apparatus OLGA [12]. $\mathrm{BhO}_{3} \mathrm{Cl}$ was synthesized in a chlorinating gas $\left(\mathrm{HCl} / \mathrm{O}_{2}\right)$ which was observed to be volatile in fused silica tubes at about $150^{\circ} \mathrm{C}$. Six detected atoms of the short-lived isotope ${ }^{266} \mathrm{Bh}$ were sufficient to proof that Bh behaves like a typical member of group 7 of the periodic table $[7,8]$. 


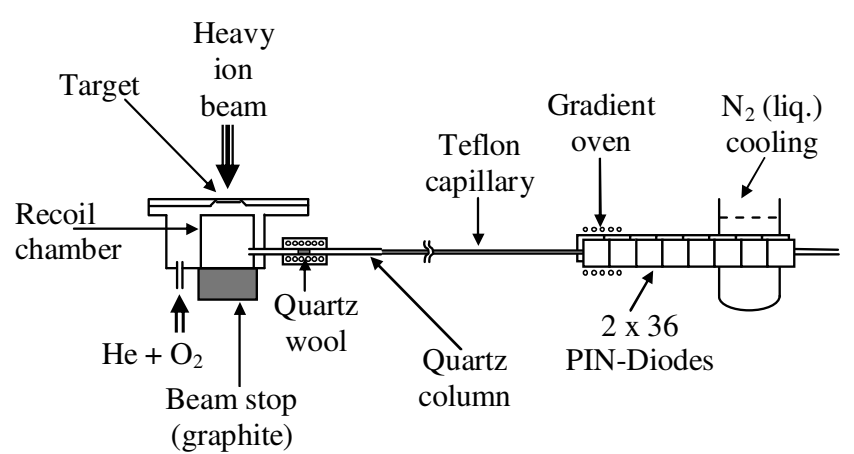

Fig. 1. IVO set-up applied in the first chemical investigation of hassium (Hs, $Z=108$ ) (for details see text).

This overview summarizes the recent hassium experiment and presents a status on the 112 experiments. In addition, two examples are highlighted how chemistry may help to confirm or refute discovery claims from physics experiments. These are the discovery claims of elements 112 and 115. In the first case it was possible to position the decay chain of ${ }^{277} 112$ at atomic number 108 (Hs) and in the second case the decay chain of ${ }^{288} 115$ at atomic number $105(\mathrm{Db})$ or its possible EC decay product element 104 (Rf), respectively.

\section{Chemical investigation of hassium (Hs)}

If Hs behaves like a normal group- 8 member of the periodic table it should form with oxygen a very volatile molecule, $\mathrm{HsO}_{4}$. For the next homologue of the same group, Os, it is well known that on a single molecule level and at ambient conditions $\mathrm{OsO}_{4}$ behaves like a gas. In macroamounts $\mathrm{OsO}_{4}$ is a liquid. To search for a volatile $\mathrm{HsO}_{4}$ a novel technique was developed, the In situ Volatilization and On-line detection device IVO [13]. From a thin-target recoiling fusion products were thermalized in oxygen containing carrier gas $(\mathrm{He})$ in order to form in situ the volatile tetroxide molecule (fig. 1).

In a continuous mode the gas was then flushed through the recoil chamber and fed into an oven kept at about $800{ }^{\circ} \mathrm{C}$ in order to retain possible particle-bound contaminants but also to rapidly oxidize $\mathrm{Hs}$ oxides from a lower than $8^{+}$oxidation state to $\mathrm{HsO}_{4}$. The gas was then injected into a channel formed by an array of 36 detector pairs mounted in form of 12 triplets at a distance of $1.5 \mathrm{~mm}$ between opposite detectors. The detectors were silicon PIN diodes that enable $\alpha$ and fission fragment spectroscopy. Along the detector pairs a stationary temperature gradient between -20 and $-175^{\circ} \mathrm{C}$ was established. The chemical information evolved from the determination of the detector pair at which the $\mathrm{HsO}_{4}$ molecules deposited, which in turn defines a deposition temperature on the surface (silicon nitride). The measurement of deposition temperatures of single atoms/molecules along a stationary negative temperature gradient is known as thermochromatography. From measured deposition temperatures adsorption enthalpies may be obtained applying

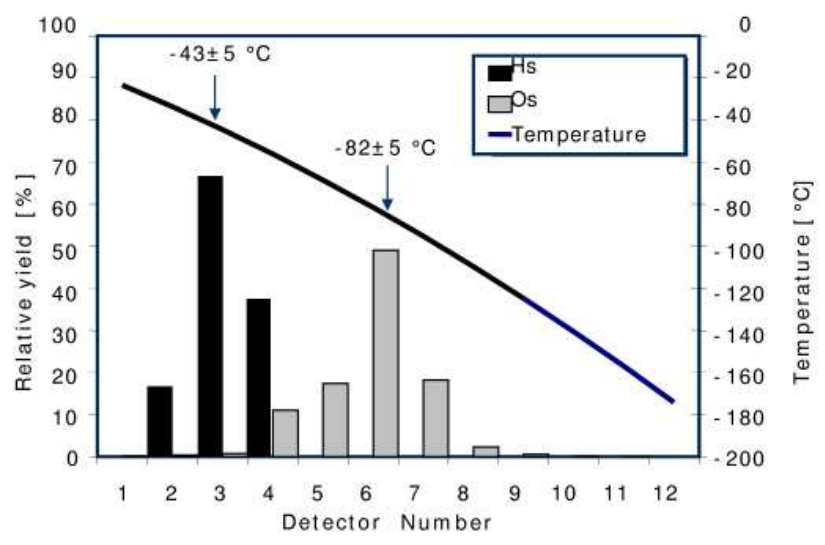

Fig. 2. Distribution of hassium and osmium tetroxide inside of the detector array. The hassium distribution resembles the behaviour of 7 atoms of ${ }^{269,270} \mathrm{Hs}$.

Monte Carlo models. From such enthalpies sublimation enthalpies may be deduced [14].

Figure 2 depicts the results of the first chemical investigation of hassium using the $10 \mathrm{~s}{ }^{269} \mathrm{Hs}$ [9]. This isotope was produced in the ${ }^{248} \mathrm{Cm}\left({ }^{26} \mathrm{Mg} ; 5 \mathrm{n}\right){ }^{269} \mathrm{Hs}$ reaction at an energy of $145 \mathrm{MeV}$ in the middle of the $0.55 \mathrm{mg} / \mathrm{cm}^{2}$ thick target. A beam dose of $1 \times 10^{18}$ particles was accumulated on the target. Seven decay chains were observed that could be assigned to ${ }^{269} \mathrm{Hs}$ and to the new nuclide ${ }^{270} \mathrm{Hs}$. The 7 observed decay chains form a clear deposition peak of $\mathrm{HsO}_{4}$ at $-43^{\circ} \mathrm{C}$. Before and after the experiment a $0.8 \mathrm{mg} / \mathrm{cm}^{2}$ thick ${ }^{152} \mathrm{Gd}$ target was bombarded with the same beam to study the chemical behaviour of osmium via the $\alpha$-decaying ${ }^{172}$ Os under otherwise identical conditions. The deposition peak of $\mathrm{OsO}_{4}$ was found at $-82^{\circ} \mathrm{C}$. This indicates a slightly stronger interaction of $\mathrm{HsO}_{4}$ with the detector surface compared to that of $\mathrm{OsO}_{4}$. Using an empirical correlation established between the adsorption properties of oxides and their volatility [14] a lower volatility of $\mathrm{HsO}_{4}$ compared to $\mathrm{OsO}_{4}$ can be deduced. Theoretical calculations predicted similar volatilities of $\mathrm{HsO}_{4}$ and $\mathrm{OsO}_{4}$. The errors of the calculations, however, are larger than the observed small difference $[15,16]$. To conclude, hassium behaves as an oddly normal member of group 8 !

\section{Chemical investigation of element 112}

Element 112 is predicted to exhibit rather exceptional chemical properties: due to relativistic effects on its filled $7 s^{2} 6 d^{10}$ electronic configuration a gaseous behaviour was predicted [17]. Also conventional thermodynamic extrapolations point to a likely behaviour as a very volatile noble metal [18]. More recent calculations corroborate these expectations $[14,19]$.

In a first chemical attempt performed at FLNR Dubna the similarity of element 112 to $\mathrm{Hg}$, its homologue in the periodic table was investigated. The experiment used the 5 min SF isotope ${ }^{283} 112$ found in the ${ }^{48} \mathrm{Ca}+{ }^{283} \mathrm{U}$ reaction at an energy of $231 \mathrm{MeV}$ using the energy filter VASSILISSA [20]. From a $2 \mathrm{mg} / \mathrm{cm}^{2}$ thick ${ }^{238} \mathrm{U}$ target 
recoiling products were thermalized in pure helium and then continuously transported along a $25 \mathrm{~m}$ long capillary to an array of 8 PIPS detectors covered with a thin layer of $\mathrm{Au}$ followed by a gas ionisation chamber (fig. 3). The separation time was up to $20 \mathrm{~s}$. Both detectors were positioned inside an array of ${ }^{3} \mathrm{He}$ neutron counters to assay prompt neutrons. During the experiment a beam dose of $2.8 \times 10^{18}{ }^{48} \mathrm{Ca}$ particles was collected at an energy of $233 \mathrm{MeV}$ in the middle of the target. Eight events were detected in the gas ionisation chamber in coincidence with 1 to 3 neutrons [10]. No SF events were detected in the PIPS detectors, where ${ }^{185} \mathrm{Hg}$ was quantitatively adsorbed that was produced simultaneously by a small admixture of $35 \mu \mathrm{g} / \mathrm{cm}^{2}{ }^{\text {nat }} \mathrm{Nd}$ deposited on top of the target. The resulting cross-section of $2 \mathrm{pb}$ agreed reasonably well with published data (4 pb [20]). It was concluded that element 112 exhibits a clearly different behaviour from $\mathrm{Hg}$, obviously interacting weaker with $\mathrm{Au}$. In a subsequent experiment, using the same reaction the thermochemical behaviour of element 112 was investigated applying the IVO device (see fig. 1) but with one side of the detector array consisting of a $\mathrm{Au}$ surface ( $2 \pi$ counting geometry). The separation time was approximately $25 \mathrm{~s}$. A $1.6 \mathrm{mg} / \mathrm{cm}^{2}$ thick ${ }^{238} \mathrm{U}$ target covered by $22 \mu \mathrm{g} / \mathrm{cm}^{2}{ }^{\text {nat }} \mathrm{Nd}$ was bombarded with $2.8 \times 10^{18}{ }^{48} \mathrm{Ca}$ particles of $231 \mathrm{MeV}$ (middle of the target). Seven single events with energies larger than $40 \mathrm{MeV}$ were detected at the position of radon, in addition to 4 additional events scattered along the detector array. The background count-rate amounted to $\sim 3$ events for the corresponding counting time. The average energies of the 7 events were lower than expected, possibly caused by ice formation on the detector surfaces below approximately $-90^{\circ} \mathrm{C}$. Therefore, this result was interpreted as an indication of a gaseous behaviour of element 112 [11]. The ambiguity of this result made a confirmation necessary with an improved set-up that separated volatile products within $2.2 \mathrm{~s}$ and operated in a $4 \pi$ counting mode, to assay SF coincidences. During an additional beam time $1.4 \times 10^{18}{ }^{48} \mathrm{Ca}$ particles (i.e. $50 \%$ of the beam dose of the first experiment) where applied to the same $1.6 \mathrm{mg} / \mathrm{cm}^{2}$ thick ${ }^{238} \mathrm{U}$ target. No SF coincidences were observed at an expected rate of $\sim 3$ events compared to the first experiment [21].

Further investigations are mandatory, especially since additional physics experiments aiming at a confirmation of the long-lived SF-activity failed. At LBNL no evaporation residue formation was observed in the ${ }^{48} \mathrm{Ca}+{ }^{238} \mathrm{U}$ reaction at a cross-section limit of about $1 \mathrm{pb}$ using the BGS separator [22]. At FLNR with the gas filled magnetic separator also no indication of a long-lived SF isotope was found but a $4 \mathrm{~s} \alpha$-emitter was discovered with a decay energy of $9.52 \mathrm{MeV}$ followed by a SF-decaying product with $T_{1 / 2}=0.18 \mathrm{~s}$. The maximum production cross-section was $2.5 \mathrm{pb}$ at an energy of $234 \mathrm{MeV}$ [23]. The second IVO experiment described above was designed to also detect such decay chains. A preliminary analysis showed that the nonobservation of any $9.5 \mathrm{MeV} \alpha$-SF correlation yields a crosssection limit of $\sim 2 \mathrm{pb}$ for a 4 s-product $(95 \%$ confidence level) [21]. Hence, presently the situation concerning the

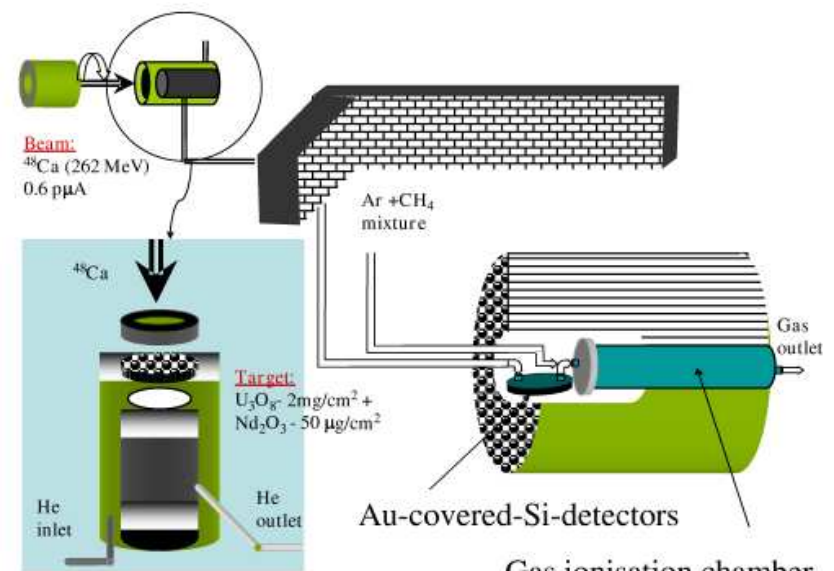

Gas ionisation chamber

Fig. 3. Set-up used for the first chemical attempt to study element 112 (from ref. [10]).

formation of element 112 in the ${ }^{48} \mathrm{Ca}+{ }^{238} \mathrm{U}$ reaction and its chemical behaviour remains very controversial.

\section{Confirmation of element discoveries by chemical means}

Chemical procedures enable the separation of a given "atomic number". This is especially easy in cases where the element to be isolated exhibits exceptional chemical properties. Such an example is hassium. The heavy members of group 8 of the periodic table (osmium and hassium) form very stable and volatile molecules with oxygen. None of the neighbouring elements show such a behaviour. In the chemistry experiment on hassium mentioned above, out of the 7 detected decay chains 3 originated from ${ }^{269} \mathrm{Hs}$ that decayed after two $\alpha$-emissions to ${ }^{261} \mathrm{Rf}$ with unexpected decay properties: 2 atoms of ${ }^{261} \mathrm{Rf}$ decayed via $\alpha$-emission with $E=8.50 \mathrm{MeV}$ and lifetimes of 0.9 and $2.4 \mathrm{~s}$, respectively, and one atom of ${ }^{261} \mathrm{Rf}$ by spontaneous fission after a lifetime of $7.90 \mathrm{~s}$ [24]. This decay pattern corroborates the observation made in the discovery experiments of element 112 , where ${ }^{261} \mathrm{Rf}$ as member of the decay chain beginning with ${ }^{277} 112$ also showed such an unexpected behaviour [4, 5]. If directly produced in a hot fusion reaction, ${ }^{261} \mathrm{Rf}$ decays with a $\alpha$-energy of $8.28 \mathrm{MeV}$ [25] and a lifetime of $113 \mathrm{~s}$ [26].

More recently, attempts were made to confirm the decay chains found at FLNR in a ${ }^{48} \mathrm{Ca}$ bombardment of ${ }^{243} \mathrm{Am}$ that yielded evidence for the formation of ${ }^{288} 115$ [2]. Three decay chains of this isotope were found, ending in a very long-lived $\mathrm{SF}{ }^{268} \mathrm{Db}$ with a half life of $16_{-6}^{+19} \mathrm{~h}$, an ideal case for a chemical confirmation!

A $1.2 \mathrm{mg} / \mathrm{cm}^{2}$ thick ${ }^{243} \mathrm{Am}$ target was bombarded with ${ }^{48} \mathrm{Ca}$ ions at an energy of $247 \mathrm{MeV}$ (middle of target) [27]. Recoiling products were collected in a thick water cooled $\mathrm{Cu}$ catcher positioned behind the target at an angle of \pm 12 degree, to suppress collection of transfer products. Once a day, after having accumulated typically $2-3 \times 10^{17}$ particles on the target, approximately $10 \mu \mathrm{m}$ of the front 
Table 1. Measured events in the $\mathrm{Db} / \mathrm{Rf}$ fraction from the chemical study of the ${ }^{48} \mathrm{Ca}+{ }^{243} \mathrm{Am}$ reaction (data from [28]).

\begin{tabular}{|c|c|c|c|c|c|}
\hline Sample & $\begin{array}{c}t_{\text {irr }}(\mathrm{a}) \\
(\mathrm{h})\end{array}$ & Beam dose & $\begin{array}{l}E_{\mathrm{bot}}+E_{\mathrm{top}}+\mathrm{Nn}^{(\mathrm{b})} \\
(\mathrm{MeV})(\mathrm{MeV})\end{array}$ & $\begin{array}{l}t_{\text {detect }}(\mathrm{c}) \\
(\mathrm{h})\end{array}$ & $\begin{array}{c}t_{\text {meas }}(\mathrm{d}) \\
(\mathrm{h})\end{array}$ \\
\hline 1 & 20 & $2.5 \cdot 10^{17}$ & $120+126+2$ & 20 & 429 \\
\hline 2 & 22 & $3.7 \cdot 10^{17}$ & $-+86+1$ & 74 & 186 \\
\hline 3 & 22 & $3.4 \cdot 10^{17}$ & $\begin{array}{l}131+124+1 \\
116+122+2\end{array}$ & $\begin{array}{l}15 \\
72\end{array}$ & 385 \\
\hline 4 & 22 & $2.9 \cdot 10^{17}$ & $\begin{array}{r}104+120+1 \\
97+125+1 \\
100+128+1\end{array}$ & $\begin{array}{l}22 \\
29 \\
51\end{array}$ & 358 \\
\hline 5 & 38 & $6.7 \cdot 10^{17}$ & $\begin{array}{r}117+118+2 \\
108+107+3 \\
110+104+0 \\
-\quad+76+2\end{array}$ & $\begin{array}{c}6 \\
9 \\
15 \\
68\end{array}$ & 861 \\
\hline 6 & 23 & $3.9 \cdot 10^{17}$ & $120+114+2$ & 39 & 933 \\
\hline 7 & 22 & $3.6 \cdot 10^{17}$ & - & - & 957 \\
\hline 8 & 45 & $7.4 \cdot 10^{17}$ & $\begin{array}{r}119+110+2 \\
118+105+2 \\
65+58+3\end{array}$ & $\begin{array}{c}5 \\
93 \\
174\end{array}$ & 910 \\
\hline
\end{tabular}

(a) $t_{\text {irr }}$ : irradiation time.

(b) $E_{\text {bot }}, E_{\text {top }}$ : measured energies in the bottom and top detectors, respectively.

(c) $t_{\text {detect }}$ : time at which event was registered after start counting.

(d) $t_{\text {meas }}$ : measuring time.

surface was removed mechanically. These Cu chips (100 to $150 \mathrm{mg}$ ) were chemically processed for dubnium and rutherfordium, with special efforts to achieve high decontamination factors from actinides [28]. Final samples were deposited on very thin organic foils and assayed for fission fragment energies in a $4 \pi$ counting geometry using silicon detectors. The counting chambers were positioned inside an array of ${ }^{3} \mathrm{He}$ counters to detect prompt neutrons. All samples were measured up to 957 hours. In 8 samples, representing a total beam dose of $3.4 \times 10^{18}$ particles, 15 fission events were observed in coincidence with up to 3 neutrons (table 1 ). The measured fission fragment energies point to a highly symmetric fissioning nucleus. After corrections of the measured kinetic energies for ionisation defect in the semiconductor detector and energy loss in the sample a total kinetic energy TKE of approximately $235 \mathrm{MeV}$ results. Taking into account a $40 \%$ detection efficiency for single neutrons a neutron multiplicity of 4.2 [27] is found. Such a signature (high TKE and $\left.\nu_{\text {tot }}\right)$ yields strong evidence for a heavy nucleus as fissioning source, far beyond, e.g. SF ${ }^{252} \mathrm{Cf}$. Therefore, the proposed assignment to ${ }^{268} \mathrm{Db}$ or ${ }^{268} \mathrm{Rf}$ from the EC-decay of ${ }^{268} \mathrm{Db}$ appears very likely. It should be mentioned that formation of transactinides in measurable amounts from the ${ }^{48} \mathrm{Ca}+{ }^{243} \mathrm{Am}$ reaction through transfer channels is extremely unlikely. In ${ }^{48} \mathrm{Ca}$ induced reactions with the actinide target ${ }^{248} \mathrm{Cm}$ the nucleon flow was observed to occur predominantly from the target to the projectile and not vice versa at energies close to the fusion barrier [29].

\section{References}

1. See e.g. S. Hofmann, Properties and synthesis of superheavy elements, in The Chemistry of Superheavy Elements, edited by M. Schädel (Kluwer Academic Publ., Dordrecht, 2003).

2. Yu.Ts. Oganessian et al., Phys. Rev C. 69, 021601 (2004).

3. K. Morimoto, Workshop on Recoil Separators for Superheavy Element Research, 27 August 2004, GSI Darmstadt, Germany.

4. S. Hofmann et al., Z. Phys. A 354, 229 (1996).

5. S. Hofmann et al., Eur. Phys. J. A 14, 147 (2002).

6. Yu.Ts. Oganessian et al., Phys. Rev C 69, 054607 (2004) and references therein.

7. R. Eichler et al., Nature 407, 63 (2000).

8. H.W. Gäggeler, A. Türler, Gas-phase chemistry, in The Chemistry of Superheavy Elements, edited by M. Schädel (Kluwer Academic Publ., Dordrecht, 2003).

9. Ch.E. Düllmann et al., Nature 418, 859 (2002).

10. A.B. Yakushev et al., Radiochim. Acta 91, 433 (2003).

11. H.W. Gäggeler et al., Nucl. Phys. A 734, 208 (2004); S. Soverna, Attempt to chemically characterize element 112, PhD Thesis, Bern University, December 2004.

12. H.W. Gäggeler et al., Nucl. Instrum. Methods A 309, 201 (1991).

13. Ch.E. Düllmann et al., Nucl. Instrum. Methods A 479, 631 (2002).

14. B. Eichler and R. Eichler, Gas-phase adsorption chromatographic determination of thermochemical data and empirical methods for their estimation, in The Chemistry of Superheavy Elements, edited by M. Schädel (Kluwer Academic Publ., Dordrecht, 2003). 
15. V. Pershina et al., J. Chem. Phys. 115, 792 (2001).

16. Ch.E. Düllmann et al., J. Phys. Chem. B 106, 6679 (2002).

17. K. Pitzer, J. Chem. Phys. 63, 1032 (1975).

18. B. Eichler, Kernenergie 19, 307 (1976).

19. V.G. Pershina, Chem. Rev. 96, 1977 (1999).

20. Yu.Ts. Oganessian et al., Eur. Phys. J. A 19, 3 (2004) and references therein.

21. R. Eichler et al., to be submitted to Radiochim. Acta.

22. W. Loveland et al., Phys. Rev. C. 66, 044617 (2002); K.E. Gregorich, private communication.
23. Yu.Ts. Oganessian et al., Phys. Rev. C 70, 064609 (2004).

24. A. Türler et al., Eur. Phys. J. A 17, 505 (2003).

25. Y. Lazarev et al., Phys. Rev. C 62, 064307 (2000).

26. A. Türler et al., Phys. Rev. C 57, 1648 (1998).

27. S.N. Dmitriev et al., Mendeleev Commun. 15, 1 (2005).

28. D. Schumann et al., submitted to Radiochim. Acta (2005).

29. H. Gäggeler et al., J. Less-Common. Metals 122, 433 (1986). 